FORUM EMPRESARIAL

Vol. 19. Núm. 2 • invierno 2014

\title{
Las técnicas financieras para la determinación de valor razonable, su aplicación en los estados financieros y las posibles repercusiones para las empresas
}

\author{
Blanca Iris Vega Castro / blanca.vega@upr.edu \\ Universidad de Puerto Rico, Recinto de Ponce \\ Pedro González Cerrud / drgonzalezcerrud@yahoo.com \\ Universidad de Puerto Rico, Recinto de Río Piedras
}

Recibido: 21 de febrero de 2013

Aceptado: 12 de junio de 2014

$\oplus$

RESUMEN

Este artículo examina la reciente normativa contable internacional, emitida por IASB y FASB, que hace referencia a la medición y divulgación del valor razonable. Se identifican las técnicas de medición aplicadas y los enfoques adoptados por empresas en diferentes países del mundo. Se analiza la aplicación de los criterios y enfoques de medición recientemente sugeridos para la determinación del valor razonable en los estados financieros y las posibles repercusiones que esto puede tener para las empresas.

Palabras clave: normas internacionales de contabilidad, valor razonable, estados financieros

\section{ABSTRACT}

This paper examines recent international accounting standards, issued by the IASB and FASB, which refer to the measurement and disclosure of fair value. We identify measurement techniques and approaches applied by companies in different countries. We also analyze the application of measurement criteria and approaches recently suggested for determining fair value in financial statements and the possible implications for businesses.

Keywords: international accounting standards, fair value, financial statements 
Vega Castro / González Cerrud

\section{INTRODUCCIÓN}

La aplicación de los conceptos de objetividad y relevancia en la preparación de los estados financieros crean lo que se conoce como el modelo mixto de contabilidad, con algunas partidas aplicables a costo histórico y otras a valor razonable. Recientemente, tanto reguladores nacionales como internacionales han ido extendiendo la aplicación del criterio de valor razonable a grupos cada vez mayores de activos y pasivos.

Varios factores han contribuido a una mayor aplicación del valor razonable; pero quizás la razón primordial para su uso se fundamenta en que éste informa mejor las características de riesgo y rendimiento reportadas en los estados financieros de las empresas. El valor razonable puede proporcionar información más relevante a los usuarios de los estados financieros, pero también menos confiable. Además, la normativa del valor razonable puede ser muy compleja y difícil de aplicar.

El impacto principal de las guías para la determinación del valor razonable es sobre el estado de situación, pero también se impactan el estado de ingresos y gastos (estado de resultados) y el estado de flujos de efectivo; por lo tanto, es importante para los preparadores, los usuarios y los auditores de los estados financieros tener claro los diversos criterios y enfoques de medición del valor razonable. En la medida en que haya validez y confianza en los datos, de esa misma forma los informes financieros serán de mayor calidad y, por consiguiente, mejor será la asignación de los recursos en la economía.

Este artículo examina la reciente normativa contable internacional, emitida por la International Accounting Standard Board (IASB) y la Financial Accounting Standard Board (FASB), que hace referencia a la medición y divulgación del valor razonable. Se identifican las técnicas de medición aplicadas y los enfoques adoptados por empresas en diferentes países del mundo. Se analiza la aplicación de los criterios y enfoques de medición recientemente sugeridos para la determinación del valor razonable en los estados financieros y las posibles repercusiones que esto puede tener para las empresas. 


\section{VALOR RAZONABLE Y LA NORMATIVA CONTABLE INTERNACIONAL}

Ante la importancia y preferencia por el uso del valor razonable como criterio de medición, los organismos que rigen la contabilidad a nivel global han requerido su uso. Tanto la FASB como la IASB han emitido estándares para ayudar al preparador y al usuario de los estados financieros en la medición e interpretación del valor razonable.

El concepto de valor razonable aparece en la literatura contable anglosajona desde hace más de 20 años. En septiembre de 2006 se emitió el pronunciamiento de la FASB núm. 157, Fair Value Measurements, actualmente conocido como la FASB Accounting Standards Codification 820, Fair Value Measurements and Disclosures (FASB, 2010). El Tópico 820 de la nueva codificación de los principios de contabilidad generalmente aceptados en Estados Unidos (USGAAP) ahora es la única guía sobre cómo las empresas deben medir y divulgar el valor razonable en sus estados financieros; y aplica a cualquier pronunciamiento de la FASB que permita o requiera el uso del valor razonable (Zyla, 2012).

También, la opción o el requisito de medir partidas aplicables al valor razonable ha estado presente de forma importante en las normas internacionales de la IASB. Tanto las Normas Internacionales de Información Financiera (NIIF) como las Normas Internacionales de Contabilidad (NIC) hacen referencia al valor razonable; pero los criterios que se utilizan para medirlo no son consistentes. No existía una norma que definiese el valor razonable para todos los elementos financieros y no financieros.

En 2008, la IASB responde a la crisis financiera global - ya que se había acusado a la implementación de las reglas contables de haberla exacerbado-, con una nueva orientación sobre la medición del valor razonable y la aceleración de los proyectos sobre éste (IASB, 2008). La normativa contable internacional, bajo el proyecto de convergencia de la FASB y la IASB, trabajó uniformando los criterios de medición del valor razonable, quizás entendiendo que de esa manera podían contribuir a la recuperación del sistema financiero internacional (FASB, 2008; SEC, 2008). 
En 2011, la IASB emitió la NIIF 13, Medición del Valor Razonable, casi idéntica al Accounting Standards Codification 820, con fecha efectiva del 1 de enero de 2013 (IFRS, 2013; Deloitte, 2013). Esta norma remplaza — mediante un solo estándar- la orientación sobre la medición del valor razonable contenida en la literatura contable existente, define valor razonable, proporciona orientación sobre cómo determinar el valor razonable y requiere revelaciones acerca de las mediciones del valor razonable.

\section{VALOR RAZONABLE}

El valor razonable es definido como el precio que se recibiría al vender un activo o se pagaría al transferir un pasivo en una transacción ordenada entre los participantes del mercado en la fecha de medición (FASB, 2012b). La norma define valor razonable a base del precio de salida, lo que implica tener una visión diferente en la forma de medir y comprender la información financiera. Otros conceptos relevantes en la definición lo son la transacción ordenada, es decir, sin presiones, en condiciones normales e independientes; y los participantes, compradores y vendedores deben ser independientes y conocedores. También resulta fundamental el mercado principal, donde se supone que se da la transacción, donde se vendería el activo o se transferiría el pasivo con el mayor volumen y nivel de actividad; y el mercado más ventajoso, aquel que maximice lo que se recibiría por el activo o minimice la cantidad que se pagaría por transferir el pasivo.

\section{TÉCNICAS DE MEDICIÓN}

En esencia, para la determinación del valor razonable, la normativa contable internacional recomienda técnicas de valoración que sean consistentes con el enfoque de costo, el enfoque de mercado y el enfoque de ingreso (FASB, 2012a). Las técnicas utilizadas dependerán de las características, circunstancias y disponibilidad de la información. 


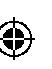

LAS TÉCNICAS FINANCIERAS PARA LA DETERMINACIÓN DE VALOR RAZONABLE...

El enfoque de costos se fundamenta en la cantidad que en la actualidad se requeriría para remplazar la capacidad de servicio de un activo, conocida como el costo de remplazo actual. Desde la perspectiva de un participante del mercado (vendedor), el precio que sería recibido por el activo es determinado basado en el costo para el participante del mercado (comprador) de adquirir o construir un activo sustituto de utilidad comparable, ajustado por obsolescencia. La obsolescencia cubre deterioro físico, obsolescencia funcional (tecnológica) y obsolescencia económica o externa.

El enfoque de mercado usa precios y otra información relevante generada por transacciones del mercado de activos o pasivos idénticos o similares. Por lo regular, se utilizan múltiplos de transacciones comparables, por lo que el valuador debe utilizar factores cuantitativos y cualitativos para determinar qué múltiplo en el rango se utilizará como comparable.

El enfoque de ingresos convierte cantidades futuras (flujos de efectivo o de ganancias) en valor presente (descontadas). La medición está basada en el valor indicado por las expectativas actuales del mercado acerca de las cantidades futuras. En el caso de activos de naturaleza durable, sería la capacidad del activo para generar flujos de efectivo durante su vida útil.

Luego de evaluar las técnicas, para aumentar la constancia y comparabilidad en el uso del valor razonable, las empresas siguen una jerarquía que provee guías sobre cómo determinarlo.

\section{JERARQuía DE CRITERIOS PARA LA DETERMINACIÓN DEL VALOR RAZONABLE}

La norma señala que se requieren datos de entrada para preparar los supuestos para la determinación del valor razonable (FASB, 2012a). Es importante resaltar que estos supuestos se hacen desde la perspectiva de los participantes de mercado y no desde el punto de vista de la entidad que reporta. Los datos de entrada observables se pueden verificar en el mercado y, por lo tanto, pueden ser verificados por terceras personas. Los datos de entrada no observables son aquéllos particulares a la entidad misma. La diferencia en la verificabilidad de los datos de entrada se refleja en 
la jerarquía establecida en esta norma, según los datos de entrada utilizados en las técnicas de valoración.

La clasificación dentro de la jerarquía depende de la naturaleza del instrumento, de la información del mercado y del juicio profesional. Se distinguen tres niveles que deben ser divulgados en las notas de los estados financieros. En el primer nivel se incluye la más alta jerarquía que da prioridad a los datos de entrada observables que reflejan precios cotizados (no ajustados), en los mercados activos (mercados en los cuales las transacciones para el activo o pasivo ocurren con suficiente frecuencia y volumen), para activos o pasivos idénticos a los cuales la entidad puede tener acceso durante la fecha de medición.

En el segundo nivel encontramos precios cotizados para activos o pasivos similares en mercados activos, o bien precios cotizados para activos o pasivos idénticos o similares en mercados que no son activos. En la menor jerarquía encontramos el tercer nivel que comprende los datos de entrada no observables para el activo o el pasivo, los supuestos de la entidad que reporta.

Se enfatiza que las técnicas de divulgación usadas para medir el valor razonable deben maximizar el uso de los datos de entrada observables y minimizar el uso de los no observables. También requiere divulgación por nivel, la cual dependerá de la naturaleza de la medición y el nivel en el cual se clasifica.

\section{Aplicación del valor Razonable}

Para conocer cómo las empresas están aplicando el valor razonable, se examinaron las notas de los estados financieros para los años 2007 al 2011, de 98 compañías alrededor del mundo (28 de Europa, 26 de Asia, 27 de Estados Unidos y 17 de Latinoamérica). El listado de países donde se aplican normas internacionales de contabilidad se obtuvo de www.iasplus.com; el nombre de las compañías de Europa y Asia se hizo en www.adir.com; y los estados financieros de cada una de las compañías se obtuvieron de www. google.com, http://finance.yahoo.com y http://finance.google. com. En el caso de las empresas de Estados Unidos y Latinoamérica, 
se utilizó la base de datos disponibles en www.alacrastore.com y la página de internet de cada una de las compañías.

Estas empresas provienen de diversas industrias, aunque en el caso de las de Estados Unidos hay una alta concentración en la industria bancaria. La gran mayoría de estas empresas tienen como auditores a las cuatro firmas más grandes (Big Four) y en los casos de las empresas de Europa y Estados Unidos, la mayoría se cotiza en la bolsa de valores.

\section{Hallazgos}

Los datos recopilados en este estudio se utilizaron para conocer las técnicas específicas que utilizan las empresas en la determinación del valor razonable y clasificarlas en uno de los tres enfoques recomendados por la normativa contable. Los resultados se presentaron por área geográfica debido a las diferencias en los niveles de la aplicación de normativas contables, de las etapas del desarrollo de los mercados de capital y entre los motores principales de crecimiento económico. Estados Unidos se separó de las otras áreas geográficas debido al tamaño y la sofisticación de su economía. A continuación se resumen las técnicas y enfoques utilizados por estas empresas, según el tipo de activo o pasivo.

La Tabla 1 (en la próxima página) presenta las partidas financieras a las que las empresas en las diferentes áreas geográficas les aplican el valor razonable para los años 2007 al 2011. Como se puede observar en ella, el uso más frecuente del valor razonable fue para instrumentos financieros, compensación en acciones, alquileres, intangibles y planes de pensiones.

Instrumentos financieros. En el caso de los instrumentos financieros se utiliza primordialmente el precio de mercado para aquellos casos donde hay un mercado activo. En el caso de los activos se utiliza el precio al que se vendería y en el caso de los pasivos se utiliza el precio al que se compraría, según reportados por un intermediario. Si no hay un activo idéntico, se utilizan comparables de activos similares; si no existe un mercado activo, se utilizan métodos de valoración como el flujo de efectivo descontado. Se utilizan 
Vega Castro / González Cerrud

Tabla 1

Partidas financieras a las que aplican valor razonable-Frecuencia por área geográfica

\begin{tabular}{lccccc}
\hline & $\begin{array}{c}\text { América } \\
\text { Latina }\end{array}$ & Asia & $\begin{array}{c}\text { Estados } \\
\text { Unidos }\end{array}$ & Europa & Total \\
\cline { 2 - 6 } Instrumentos financieros & 12 & 23 & 22 & 27 & 84 \\
Intangibles & 5 & 13 & 18 & 21 & 57 \\
Compensación en acciones & 2 & 17 & 24 & 19 & 62 \\
Materia prima & 8 & 3 & 4 & 1 & 16 \\
Alquileres & 6 & 11 & 25 & 20 & 62 \\
Activos de inversión & 0 & 7 & 17 & 7 & 31 \\
Planes de pensiones & 2 & 10 & 25 & 14 & 51 \\
Combinación de empresas & 8 & 3 & 14 & 22 & 47 \\
Concesiones de gobierno & 0 & 3 & 8 & 4 & 15 \\
Propiedad planta y equipo & 8 & 0 & 0 & 0 & 8 \\
\hline
\end{tabular}

Fuente: Elaboración propia

diversas tasas para descontar los flujos de efectivo, como por ejemplo: la tasas libres de riesgo, ajustadas por los riesgos particulares al activo o pasivo (conocidas como primas por riesgo, en la mayoría de los casos el riesgo mencionado es el riesgo de liquidez), y la tasa efectiva de rendimiento original. Frecuentemente, si había una dispersión amplia de los valores razonables, se utilizaba el costo original como medida de valoración; es decir, no había confianza en los valores determinados.

Deudas a largo plazo. En el caso de la deuda a largo plazo, se valora el valor presente de los pagos futuros acordados contractualmente, descontados de una tasa que reflejará los spread por riesgo de crédito de la deuda y el punto equivalente en la curva de rendimiento; en otros casos, se utiliza la tasa de interés corriente disponible al grupo empresarial, independientemente de los vencimientos y las características propias de cada deuda. 
LAS TÉCNICAS FINANCIERAS PARA LA DETERMINACIÓN DE VALOR RAZONABLE...

Swaps y forwards. Como parte de los instrumentos financieros, los contratos swaps y los forwards se clasifican como tasas de interés y divisas. En el caso de los swaps se utilizan los precios del mercado, los modelos de flujo de efectivo netos que se pagarían o recibirían del swap descontados a base de la curva swap. Otros utilizan comparables de swap y en algunos casos se utilizan modelos (por ejemplo, los modelos Hull-White o Black-Derman-Toy). En el caso de los instrumentos forwards, la mayoría de las entidades utilizan los precios del mercado para hacer la valoración. Otra forma muy común de valorar estos contratos forwards es descontando del valor presente los flujos contractuales estimados en la tasa corriente de mercado. Otros utilizan los servicios de brokers o emplean para contratos de menos de un año, el modelo proporcional y para más de un año, una fórmula actuarial.

Derivados. Para los derivados en general, se utiliza el precio de mercado. Si no hay un mercado activo, se usan derivados. Si no hay un mercado activo, se emplean métodos de valoración como el flujo de efectivo descontado. El modelo de Black-Scholes y el modelo binomial son modelos que se usan con alguna frecuencia; por lo tanto, es común encontrar supuestos de tasa libre de riesgo, volatilidad del precio del instrumento al que el derivado representa, matrices de correlación y vencimientos, entre otros.

Valoración de compensación en opciones sobre acciones de las empresas. En el caso de las opciones para obtener acciones como parte del plan de compensación, el método más utilizado es el método de Black-Scholes, seguido por el modelo binomial y la simulación de Montecarlo. En menor medida se utiliza el precio de la acción de la compañía al momento de otorgar la opción sobre acciones de dicha compañía. Los datos de entrada necesarios para la aplicación del modelo de Black-Scholes son: la tasa libre de riesgo (por lo regular es la tasa de rendimiento de la deuda del gobierno con igual vencimiento que el vencimiento de la opción), el precio de mercado de la acción de la compañía, el tiempo al vencimiento de la opción y la volatilidad del precio de la acción. 
Intangibles. El intangible al que se le hace más referencia en las notas a los estados financieros es la plusvalía; el cálculo típico de valor razonable se hace cuando se aplica la prueba de deterioro en el valor de este activo. Esta prueba requiere calcular el valor recobrable de la plusvalía. Para determinar el valor recobrable, se descuenta del valor presente el flujo futuro de efectivo que se espera recibir de la unidad generadora de efectivo. Por lo regular, se proyectan flujos de efectivo a un promedio de cinco años y la tasa de descuento es la tasa libre de riesgo antes del impuesto ajustada por los riesgos particulares del activo. Algunas empresas mencionaban que utilizaban los servicios de valuadores especializados en plusvalía.

Otros intangibles. Otros intangibles y sus métodos de valoración son: licencias, valoradas como el flujo de efectivo descontado ajustado por la probabilidad de que la licencia sea exitosa; relaciones con el cliente, valoradas en función del valor razonable de los contratos otorgados a cada cliente; y franquicias, valoradas por valuadores profesionales. En muchos casos, se proyectan los flujos de efectivo considerando factores tales como tasas de crecimiento y cambios en márgenes de ganancias, entre otros.

Planes de pensiones. En el caso de los planes de pensiones de beneficios definidos, se tienen que valorar tanto los activos como los pasivos del plan. El valor de los activos se fundamenta en los precios de los instrumentos que forman el portafolio de inversiones del plan, siempre y cuando exista un mercado activo para dichos instrumentos; de no existir un mercado activo, se utilizan los mismos modelos que se utilizan para los instrumentos financieros. En el caso de los pasivos del plan de pensiones de beneficios definidos, el valor se determina proyectando los beneficios futuros y se descuenta a una tasa que equivale al rendimiento de un bono libre de riesgo de crédito a un vencimiento a largo plazo. Típicamente, los datos de entrada para el cómputo del valor del pasivo son provistos por el actuario. 
LAS TÉCNICAS FINANCIERAS PARA LA DETERMINACIÓN DE VALOR RAZONABLE...

Activos de inversión. En el caso de los activos de inversión, se utiliza el valor presente de los flujos de efectivo netos esperados de los activos descontados a una tasa que refleja la tasa libre de interés más una prima por riesgo del activo. Este riesgo particular del activo es definido en la mayoría de las ocasiones como la volatilidad proyectada de los flujos de efectivo netos.

Propiedad, planta y equipo. En el caso de propiedad, planta y equipo, se utilizan los servicios de valuadores profesionales. Lo interesante de este caso en particular es que la valoración se lleva a cabo en intervalos mayores de un año; es decir, estas valoraciones tienden a hacerse cada tres años la mayoría de las veces y cada cinco años en algunos casos.

Para la combinación de negocios, se utilizan los métodos antes señalados para activos y pasivos con la opinión de valuadores profesionales que, por lo regular, se concentran en valorar activos a largo plazo no financieros (propiedad, planta y equipo, y plusvalía).

Las notas de los estados financieros, en cuanto a técnicas de valoración, son más detalladas en mercados más avanzados como Estados Unidos y Europa, y en menor grado en Asia y América Latina. Las empresas del sector financiero son las que más divulgación hacen de sus métodos y supuestos para calcular el valor razonable.

A continuación se presenta la Tabla 2, con los enfoques utilizados por las compañías por área geográfica para los años 2007 al 2011.

Tabla 2

Enfoques de valor razonable aplicado por compañias por área geográfica

\begin{tabular}{lccc}
\hline & Mercado & Ingreso & Costo \\
\hline América Latina & 6 & 17 & 12 \\
Asia & 23 & 26 & 2 \\
Estados Unidos & 26 & 27 & 2 \\
Europa & 28 & 28 & 3 \\
Total & 83 & 98 & 19 \\
\hline
\end{tabular}

Fuente: Elaboración propia 
La evidencia nos revela que el enfoque más aplicado es el de ingreso con el uso de la técnica de flujos de efectivo descontados del valor presente. También se encontró que el principal indicador de valor de mercado es el precio. La gerencia utiliza modelos más complejos para instrumentos financieros sin mercado secundario y los valuadores profesionales son utilizados para activos a largo plazo no financieros. Entre más ilíquido sea el activo, mayor es el uso de valuadores independientes especializados en el tipo de activo; es decir, se depende menos de mercados o modelos estándares.

Como era de esperarse, la aplicación de los métodos de determinación del valor razonable que no están basados en el precio del mercado, abunda más en las partidas de los estados financieros de compañías de América Latina. Esto es así por la falta de actividad y liquidez en los mercados de muchos de estos países, contrario a Estados Unidos donde existen mercados financieros más desarrollados. En Estados Unidos, la normativa afecta primordialmente los instrumentos financieros y casi en su totalidad utilizan enfoques de mercado e ingresos.

\section{IMPLICACIONES PARA LA EMPRESA}

La aplicación de los diversos enfoques recomendados para determinar el valor razonable de una partida financiera puede tener implicaciones en la administración de las empresas. Luego de examinar la reciente normativa contable internacional sobre el valor razonable y de identificar las técnicas y los enfoques adoptados por empresas en diferentes países del mundo, los autores de este estudio elaboraron una serie de implicaciones que clasificaron como internas y externas. De la revisión de la literatura no se desprende que estas implicaciones se hayan investigado antes, por lo que pudiera representar una oportunidad de investigación. A continuación, las posibles implicaciones que hemos identificado y una breve explicación. 


\section{Repercusiones internas}

- Riesgo empresarial.El impacto de la volatilidad potencial de los valores de activos, pasivos, capital y las ganancias, producto de la variabilidad periódica en los valores razonables calculados le añade una dimensión adicional al riesgo empresarial total.

- Reto ante la necesidad de un equipo de trabajo integrado. La amplia gama de disciplinas que deben interactuar para la aplicación de los enfoques de valores razonables recomendados puede representar un reto a aquellas empresas que no cuentan con el conocimiento técnico interdisciplinario.

- Aumento en costos de tecnología y personal. La estructura tecnológica necesaria para mantener y actualizar los bancos de datos con los datos de entrada para la determinación del valor razonable puede requerir altos niveles de inversión en equipo y personal.

- Ineficiencias en la composición y el vencimiento de activos y pasivos. Se debe evaluar el impacto que la distribución de vencimientos y la liquidez de activos y pasivos pueda tener sobre el monto y la calidad del estimado del valor razonable; por ejemplo, los instrumentos con vencimientos largos son más susceptibles a cambios en tasa de interés.

- Alteración de la política de inversiones. La gerencia financiera va a estar inclinada a favorecer instrumentos a corto plazo, de alta liquidez y de poco riesgo crediticio, para reducir la volatilidad del valor razonable. Esto puede afectar un criterio de mejor práctica que señale que los vencimientos de los activos deben ajustarse a los vencimientos de los compromisos (pasivos) que los mismos van a cubrir.

- Aumento en el costo del financiamiento. En la medida en que se seleccionen financiamientos a mayor plazo, más volatilidad va a tener el valor razonable de dichos pasivos debido a cambios en factores tales como las tasas de interés. La volatilidad en el valor de la deuda puede afectar negativamente el costo del 
capital marginal de la empresa, si las tasas de interés suben, o positivamente, si las tasas de interés bajan.

- Mayor adiestramiento. En el proceso de determinación del valor razonable concurren las disciplinas de contabilidad, finanzas y economía. Al ser un tema que por primera vez se está aplicando y que no pudiera formar parte de la capacitación formal de los contadores, es probable que las empresas se vean en la necesidad de desarrollar un programa de adiestramientos.

- Impacto en la determinación de la compensación a la gerencia y la asignación de recursos. Las formas de medir más utilizadas para evaluar la gestión frecuentemente utilizan como materia prima la información de los estados financieros. Si se utiliza el valor razonable para calcular muchas de estas partidas que se emplean para calcular las métricas, es probable que muchas decisiones gerenciales operacionales o financieras no se vean reflejadas en las métricas.

- Incertidumbre sobre las implicaciones de impuestos. El cambio en valor razonable va a impactar el estado de resultados y el estado de situación. Dependiendo de las leyes de impuestos de cada uno de los países, las ganancias o pérdidas producto de aumentos o disminuciones en el valor razonable reflejadas en el estado de resultados, y los cambios en los valores reflejados en el estado de situación pudieran tener impacto sobre impuestos sobre ingresos e impuestos sobre la propiedad, respectivamente.

- Implicaciones éticas. La determinación del valor razonable va a depender de la aplicación de los métodos, supuestos y datos de entrada que haga la gerencia. Estos parámetros deben ser seleccionados por la gerencia de las empresas empleando criterios únicamente profesionales, guiados por un marco de conducta ética. 


\section{RePERCUSiOnes EXTERNAS}

- Dificultad en el cumplimiento de cláusulas de financiamiento. El uso del valor razonable causa variaciones en las partidas que se consideran en algunas razones financieras computadas por los acreedores para evaluar el cumplimiento de algunas cláusulas.

- Costos de negociación de cláusulas de financiamiento. Se refiere al uso del concepto de valor razonable en el proceso de negociación de cláusulas para evaluar impago y el aumento en el costo de financiamiento debido a un desaire técnico.

- Impacto sobre el regulador. Se puede argumentar que un regulador (por ejemplo, la banca y los seguros) pudiera impactarse dependiendo si en su evaluación va a utilizar partidas que estén identificadas como valor razonable de acuerdo con la normativa contable, o si va a utilizar otro tipo de parámetro de valoración (costo histórico).

- Impacto sobre la opinión de los analistas. La comunidad de analistas de inversión utiliza, como parte de sus criterios, razones financieras que serán impactadas en menor o mayor grado por el uso del valor razonable.

- Demandas judiciales potenciales. Si la gerencia no descarga de una forma adecuada su responsabilidad de utilizar métodos, datos de entrada y supuestos adecuados al proceso de valoración, más propensa estará la empresa a que la demanden.

- Volatilidad de las ganancias. Si hay volatilidad de las ganancias debido a cambios en el valor razonable, dicha volatilidad pudiera afectar la valoración de la acción de la empresa en el mercado.

- Volatilidad en el pago de dividendos. Si una compañía tiene una política de pago de dividendos a base de un porcentaje fijo de la ganancia por acción, la volatilidad de dicha ganancia por acción va a provocar también volatilidad en los dividendos pagados. 
- Falta de calidad de los estimados. La jerarquía establecida, en función de la observación de los datos de entrada utilizados, es un mecanismo que los usuarios de los estados financieros utilizan para evaluar la calidad de los estimados de valor razonable. Una menor calidad de estimado de valor razonable pudiera interpretarse como menor calidad de los estados financieros con las consecuencias que dicha percepción tiene en la evaluación de la empresa.

- Volatilidad en los indicadores financieros. Además de la ganancia por acción, existen otros indicadores que los analistas siguen muy de cerca como, por ejemplo, el rendimiento sobre el capital. La volatilidad en los estimados de valor razonable impactan este tipo de indicadores haciéndolos más volátiles.

- Mayor escrutinio de otros grupos de presión. El valor de los activos es uno de los criterios financieros que utilizan los grupos de presión para definir su visibilidad; por lo tanto, en la medida en que el valor de los activos aumente debido a la aplicación del valor razonable, más visibles se hacen estas empresas. Este hecho podría provocar una mayor demanda de los grupos de presión para que actúen en forma socialmente responsable.

\section{Conclusiones}

El valor razonable ha cambiado la forma en que se presenta y analiza la información financiera de las empresas. Todo indica que el valor razonable, como criterio de medición de las partidas de los estados financieros, llegó para quedarse, porque es utilizado ampliamente y se espera que su uso continúe en aumento. A pesar de todas las ventajas - principalmente porque permite obtener una imagen más relevante de la posición financiera de la empresa-, la aplicación del concepto de valor razonable implica el uso del juicio profesional por parte de la gerencia financiera, lo cual puede reducir la confianza en los estados financieros. 
El hecho de que el enfoque de mercado se utilice con más frecuencia en países con mercados de capital desarrollado, como Estados Unidos, no garantiza que el precio del mercado sea el mejor indicador del valor. La crisis financiera de 2008 demostró que en mercados ilíquidos y poco ordenados, el precio transado en el mercado es más producto de la especulación que de los fundamentos. Precisamente debido a esta crisis, los entes encargados de emitir la normativa contable ajustaron dichas normas, incluyendo el requisito de que el mercado tuviese liquidez y orden.

En el caso de países en vías de desarrollo, las mayores dificultades con la aplicación del enfoque de mercado podrían ser la disponibilidad de información transaccional por un lado, lo pequeño que son los mercados de capital y la reducida actividad transaccional. Estos factores provocan la falta de liquidez y, por ende, la falta de relevancia de precios de transacciones. También, lo reducido del tamaño de los mercados de capital dificulta la aplicación de la técnica de múltiplos de mercado, porque es difícil tener una muestra representativa para escoger comparables por sector o industria. La situación deja como alternativa potencial el uso de comparables del mercado completo y no de la industria como idealmente debería ser.

Del análisis se desprende que el enfoque más aplicado es el de ingreso con el uso de flujos de efectivo descontados del valor presente. Esta técnica de valor presente de flujos de efectivo, tiene el reto no sólo de proyectar los futuros flujos de efectivo, sino de determinar la tasa de descuento que se le va aplicar, porque el cómputo de ésta es difícil particularmente en los mercados de capital menos desarrollados. A pesar de los retos en su aplicación, las compañías en regiones con mercados de capital menos desarrollados, están más propensas a utilizar los enfoques de ingreso y de costos.

La capacidad de los enfoques para que rindan la información más relevante, puede cambiar dependiendo del ciclo económico. En los años de la crisis financiera nos percatamos de cómo un mercado ilíquido y no ordenado pudo haber reducido la capacidad del enfoque de mercado frente al de costo. Por otro lado, se observa 
que la aplicación de los enfoques variará según el área geográfica. Estos cambios de la aplicación del enfoque en un país a través del tiempo y de la aplicación entre países limitarán la comparabilidad de los estados financieros.

Este estudio sugiere que la aplicación del valor razonable en la contabilidad puede tener implicaciones para las empresas, las cuales pueden ser fuente de investigaciones futuras. Los resultados de estos estudios podrían ser cada vez más trascendentes, ante la preferencia cada vez mayor por el uso del valor razonable como criterio de medición. 
LAS TÉCNICAS FINANCIERAS PARA LA DETERMINACIÓN DE VALOR RAZONABLE...

\section{Referencias}

Deloitte Touche Tohmatsu Limited. (2013). IFRS 13-Fair Value Measurement. Recuperado de http://www.iasplus.com/en/ standards/ifrs/ifrs13

Financial Accounting Standards Board (FASB). (2008). IASB and FASB announce membership of financial crisis advisory group. Recuperado de http://www.fasb.org/news/nr123008.shtml

Financial Accounting Standards Board (FASB). (2010). Statement of financial accounting standards no. 157. Recuperado de http:/ / www.fasb.org/cs/BlobServer?blobkey=id\&blobwhere= $1175823288587 \&$ blobheader=application $\% 2$ Fpdf\&blobcol $=u$ rldata\&blobtable $=$ MungoBlobs

Financial Accounting Standards Board (FASB). (2011). IASB and FASB issue common fair value measurement and disclosure requirements. Recuperado de http://www.fasb.org

Financial Accounting Standards Board (FASB). (2012a). Accounting standards codification. Recuperado de https://asc.fasb.org/ section\&trid $=6532147$

Financial Accounting Standards Board (FASB). (2012b). Master glossary. Recuperado de https:/ /asc.fasb.org/glossary\&letter=F

International Accounting Standards Board (IASB). (2008). Measuring and disclosing the fair value of financial instruments in markets that are no longer active. Recuperado de http:// www.ifrs.org/News/PressReleases/Documents/IASB_Expert_ Advisory_Panel_October_2008.pdf

International Financial Reporting Standards (IFRS). (2013). IFRS 13: Fair value measurement. Recuperado de http://www.ifrs.org/ IFRSs/IFRS-technical-summaries/Documents/English $\% 20$ Web\%20Summaries\%202013/IFRS\%2013.pdf

United States Securities and Exchange Commission (SEC). (2008). Report and recommendations pursuant to section 133 of the Emergency Economic Stabilization Act of 2008: Study on mark-tomarket accounting. Recuperado de http://www.sec.gov/news/ studies/2008/marktomarket123008.pdf

Zyla, M. (2012). Fair value measurement: Practical guidance and implementation. Hoboken, NJ: John Wiley \& Sons, Inc. 